The AstrophysicAl JouRNAL, 436:642-653, 1994 December 1

(C) 1994. The American Astronomical Society. All rights reserved. Printed in U.S.A.

\title{
EVIDENCE FOR AXISYMMETRIC HALOS: THE CASE OF IC 2006
}

\author{
MariJn Franx \\ Harvard-Smithsonian Center for Astrophysics; and Kapteyn Laboratory, P.O. Box 800, 9700 AV Groningen, The Netherlands \\ J. H. vaN GORKOM \\ Astronomy Department, Columbia University, 538 West 120th Street, New York, NY 10027 \\ AND \\ TIM DE ZEEUW \\ Sterrewacht, Huygens Laboratorium, Postbus 9513, 2300 RA Leiden, The Netherlands \\ Received 1994 February 9; accepted 1994 May 31
}

\begin{abstract}
We present a new method to derive the shape of the potential from the velocity field of a gas ring, or a gas disk with a flat rotation curve. The method is an extension of previous work by Binney and Teuben, and it can detect deviations from axisymmetry at the level of a few percent. The velocity field of the ring or disk is expanded into harmonics, and we present analytic expressions which relate these harmonic terms to the intrinsic parameters, and the viewing angles. We show that both the velocity field and the geometry of the ring are necessary to give complete information on the shape of the potential in the plane of the ring. The velocity field alone gives incomplete information for small ellipticities. We present new neutral hydrogen data on the $\mathrm{H} I$ ring around the early-type galaxy IC 2006, which was discovered by Schweizer, van Gorkom, \& Seitzer (1989). The new data show that the ring is filled and has a remarkably regular velocity field. Application of our method to this gas ring shows that the halo must be close to perfectly axisymmetric. We detect a nonsignificant ellipticity of the potential of $0.012 \pm 0.026$. The $95 \%$ confidence limit on the ellipticity is 0.05 . This implies that the potential is nearly circular in the plane of the ring. The analysis indicates that the circular velocity is nearly constant from $0.5 R_{e}$ to $6.5 R_{e}$. We confirm that the $M / L$ ratio in the outer parts increases (Schweizer et al. 1989). The stellar component probably has a strong disk. The data demonstrate that galaxies other than spiral galaxies have massive halos. The inferred shape of the halo can be contrasted to the strongly triaxial halos found in simulations of dissipationless halo formation. As suggested by Katz \& Gunn (1991), the inclusion of baryonic matter in the simulations may be necessary to resolve this issue.

Subject headings: galaxies: individual (IC 2006) — galaxies: kinematics and dynamics — galaxies: structure radio lines: galaxies
\end{abstract}

\section{INTRODUCTION}

The discovery of dark halos around spiral galaxies poses the obvious question whether elliptical galaxies and SO galaxies have similar halos. This question is fundamental to understanding the nature of dark matter and to understanding the process of galaxy formation and evolution. The presence of dark matter in clusters, which contain many early-type galaxies, demonstrates clearly that these galaxies exist in environments that are "rich" in dark matter. Nevertheless, the information on the density distribution in individual elliptical galaxies is much scarcer than the available information on spirals (e.g., Kent 1990; de Zeeuw 1992).

The matter distribution of spiral galaxies is usually inferred from extended gas rotation curves. Unfortunately, very few ellipticals show filled gas disks. Furthermore, the modeling is often uncertain because of poor signal-to-noise ratio, and the uncertainty in viewing angles. Additionally, dark halos may well have triaxial shapes (Binney 1978). If one ignores the deviation from axisymmetry, one would infer the wrong viewing angles and the wrong circular velocity. Fortunately, the triaxiality of the halo produces a characteristic signature in the velocity field of the gas (Binney 1978; Teuben 1991). Thus the velocity field itself can be used to constrain the shape of the halo.
Here we extend the previous work of Binney and Teuben, and we analyze in detail the effects of triaxial potentials on the observed velocity field. We adopt the approach introduced by Teuben, in which he expands the observed velocity field into harmonics. We apply this method to the case of a gas ring around a galaxy. We show how the harmonic expansion can be related directly to the intrinsic parameters of the ring and the galaxy.

The purpose of the analysis is twofold: we derive the shape of the galaxy potential in the ring, and, simultaneously, we derive the total mass inside the ring. We apply the method to IC 2006, an early-type galaxy in the Fornax cluster. Schweizer, van Gorkom, \& Seitzer (1989) discovered that this galaxy has a regular $\mathrm{H}$ I ring at $\sim 6.5 R_{e}$. We obtained high-resolution and high signal-to-noise ratio $\mathrm{H}$ I data at the VLA. The regular appearance of the ring allows us to put tight constraints on the deviation from axisymmetry and on the mass of the galaxy inside the ring.

We proceed in the following way. In $\S 2$ we analyze the velocity field produced by a triaxial potential. We present the method we adopt to derive the elongation of the potential from the gas kinematics. Section 3 presents the new $\mathrm{H}$ I data, which are analyzed in $\S 4$. In $\S 5$ the available optical data is modeled, and a mass model is constructed. The results are discussed in $\S 6$. 


\section{SIGNATURES OF ELONGATED POTENTIALS}

\subsection{Projection of Epicyclic Orbit}

We analyze the case where gas is moving along stable closed orbits in a triaxial potential. If the galaxy potential is near to circular and nonrotating, the cold gas is expected to move along stable closed orbits in a symmetry plane (Tohline, Simonson, \& Caldwell 1982). Shocks are not important in this case, and an equilibrium configuration can be reached. The stable closed orbits are generally elliptical and are elongated perpendicular to the elongation of the potential. Epicyclic theory provides a good approximation to the shape and velocity field of such orbits in slightly elongated potentials (Gerhard \& Vietri 1986; Binney \& Tremaine 1987). We consider the case of a gas ring, i.e., a single closed orbit.

In a potential with constant ellipticity $\epsilon_{\text {pot }}$ and circular velocity $v_{c} \propto R^{\alpha}$, the closed orbit is an ellipse to first order, with ellipticity

$$
\epsilon_{R}=1-\frac{R_{\min }}{R_{\max }}=\frac{1+\alpha}{1-\alpha} \epsilon_{\mathrm{pot}} .
$$

We assume the orbit lies in the $(x, y)$-plane and is elongated along the $y$-axis. We use $R$ and $\phi$ to denote polar coordinates defined by $x=R \cos \phi, y=R \sin \phi$ (Fig. 1a). Three viewing angles $i, \phi_{\text {obs }}$, and $\Gamma_{\text {obs }}$ specify the projection of the orbit on the sky. The angles $\phi_{\text {obs }}, i$ define the line of sight by $(\phi, \theta)=$ $\left(\phi_{\mathrm{obs}}, i\right)$, where $\phi$ and $i$ are the usual spherical polar angles. The angle $\Gamma_{\text {obs }}$ specifies the angle between the observer's coordinates in the plane of the sky and the projected $x$ - and $y$-axis (Fig. 1b).

We introduce a new coordinate along the orbit, $\psi=\phi$ $-\phi_{\text {obs }}-\pi / 2$. This coordinate is zero along the line of nodes, i.e., it is zero along the apparent major axis of the projected circular orbit $R=$ constant. As a consequence, the velocity field of a circular ring is simply given by $V_{\mathrm{rad}}=v_{c} \sin i \cos \psi$.

For an elliptic orbit, the line-of-sight radial velocity is not so easily expanded. In the Appendix we show that the radial velocity can be expressed as

$$
v_{r}=c_{1} \cos \psi+s_{1} \sin \psi+c_{3} \cos 3 \psi+s_{3} \sin 3 \psi,
$$

where

$$
\begin{aligned}
& c_{1}=\left[1-(1-a) \epsilon_{R} \cos 2 \phi_{\mathrm{obs}}\right] v_{c} \sin i, \\
& s_{1}=\left[(1-a) \epsilon_{R} \sin 2 \phi_{\mathrm{obs}}\right] v_{c} \sin i, \\
& c_{3}=\left(a \epsilon_{R} \cos 2 \phi_{\mathrm{obs}}\right) v_{c} \sin i, \\
& s_{3}=-\left(a \epsilon_{R} \sin 2 \phi_{\mathrm{obs}}\right) v_{c} \sin i,
\end{aligned}
$$
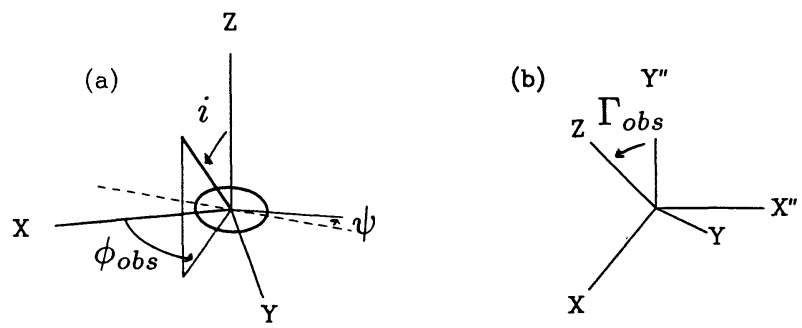

Fig. 1.- (a) Geometry of the projected orbit. The orbit is indicated by the solid ellipse. The orbit lies in the $(x, y)$-plane and is elongated along the $y$-axis. The viewing angles $i$ and $\phi_{\text {obs }}$ are equal to the polar coordinates of the line of sight. The line of nodes is indicated by the dashed line. The angle $\psi$ is the angle in the plane of the orbit and is defined to be zero at the line of nodes. $(b)$ The angle $\Gamma_{\text {obs }}$ is the position angle of the $z$-axis with respect to the observers frame. and we have written $a=\frac{1}{2} \alpha /(1+\alpha)$. This result is valid for small $\epsilon_{R}$. In the case of a flat rotation curve $(a=0)$, both $c_{3}$ and $s_{3}$ vanish, and the resulting expression,

$$
v_{r}=v_{c} \sin i\left[\left(1-\epsilon_{R} \cos 2 \phi_{\mathrm{obs}}\right) \cos \psi+\epsilon_{R} \sin 2 \phi_{\mathrm{obs}} \sin \psi\right],
$$

is valid for a full disk, if the ellipticity and inclination $i$ are constant with radius. Kuijken \& Tremaine (1994) give a number of other properties of the gas kinematics in an elongated potential.

The above expressions are obviously more complex than the formula for a circular orbit, for which $\epsilon_{R}=0$ and all terms except the $\cos \psi$ term vanish. The sizes of the additional terms $s_{1}, s_{3}$, and $c_{3}$ depend both on the internal parameters and on the viewing angles. The main effect caused by the ellipticity of the potential is the additional $s_{1} \sin \psi$ term. It depends on the viewing angle $\phi_{\text {obs }}$ : if we are looking along the major or minor axis of the ellipse, then it is zero. Along the intermediate axis $\left(\sin 2 \psi_{\text {obs }}=1\right)$, the term is maximal.

This $s_{1} \sin \psi$ term produces the two characteristic effects of noncircular potentials noted by Binney (1978): (1) the velocity gradient along the projected axis of rotation will be nonzero, and (2) the projected axis of zero radial velocity will not be perpendicular to the projected axis of maximum radial velocity. It is straightforward to show that the velocity along the projected axis of rotation equals $\left|v_{r, \text { rot }}\right|=\epsilon_{R} v_{c}\left|\sin 2 \phi_{\text {obs }}\right| \sin i$. The position angles of zero and maximum radial velocity $\Gamma_{0}$ and $\Gamma_{\max }$ are easily derived from equation (2). We note that the angle $\psi$ is related to the position angle on the sky $\Gamma$ by

$$
\tan \left(\Gamma-\Gamma_{\text {node }}\right)=\tan \psi \cos i,
$$

where $\Gamma_{\text {node }}$ is the position angle of the line of nodes. It follows that for small $\epsilon_{R}$, the position angles of zero and maximum velocity are given by

$$
\begin{gathered}
\Gamma_{0}-\Gamma_{\text {node }}=\frac{\pi}{2}+\frac{s_{1}-s_{3}}{c_{1}-3 c_{3}} \frac{1}{\cos i}=\frac{\pi}{2}+\frac{\epsilon_{R} \sin 2 \phi_{\mathrm{obs}}}{\cos i}, \\
\Gamma_{\max }-\Gamma_{\text {node }}=\frac{s_{1}+3 s_{3}}{c_{1}+9 c_{3}} \cos i=(1-4 a) \epsilon_{R} \sin 2 \phi_{\mathrm{obs}} \cos i .
\end{gathered}
$$

As a consequence, the difference between these two angles is approximately given by

$$
\Gamma_{0}-\Gamma_{\max }=\frac{\pi}{2}+\epsilon_{R} \sin 2 \phi_{\mathrm{obs}}\left[\frac{1}{\cos i}-(1-4 a) \cos i\right] .
$$

Thus, the nonperpendicularity of the kinematic axes is largest for nearly edge-on galaxies, with $\sin 2 \phi_{\text {obs }}=1$. At small inclinations (nearly face-on galaxies), the nonperpendicularity will be small. It follows that the measurement of $\Gamma_{0}-\Gamma_{\max }$ constrains only the $\epsilon_{R} \sin 2 \phi_{\mathrm{obs}}$ term, and not $\epsilon_{R} \cos 2 \phi_{\mathrm{obs}}$, or the total ellipticity. The sensitivity depends on the inclination angle $i$.

\subsection{Measuring the Ellipticity of the Potential}

Equation (2), above, can be used to fit the observed velocity field. In principle, the ellipticity $\epsilon_{\text {pot }}$, and the viewing angles, can be derived from such a fit. In practice, such a fit will often not work very well, because the parameters are highly correlated and are sometimes ill-determined. 
Here we choose another approach: we fit a circular orbit to the geometry of the ring or to the velocity field of the ring. This gives approximate viewing angles $\hat{i}, \hat{\Gamma}_{\text {obs }}$. These will generally not be equal to the true viewing angles, because the ring might be elliptical. Then we deproject the velocity field in the coordinate system defined by $\hat{i}, \hat{\Gamma}_{\text {obs }}$, and expand it into harmonics. If the ring is truly circular, then only the $\cos \hat{\psi}$ term will be significant. If the potential is elliptical, then nonzero $\sin \hat{\psi}, \cos$ $3 \hat{\psi}$, sin $3 \hat{\psi}$ terms will be measured. A similar method was introduced by Teuben (1991), who performed numerical simulations of his technique.

In the Appendix we show how the results from the harmonics expansion can be directly related to the internal parameters and the viewing angles. Two effects play a role; first, the ellipticity of the potential introduces nonzero $s_{1}, c_{3}, s_{3}$ terms when the velocity field is expanded in the correct coordinate system. The difference between the correct viewing angles $\Gamma_{\text {obs }}$, $i$ and the approximate viewing angles $\hat{\Gamma}_{\text {obs }}, \hat{i}$ will add additional terms. This changes the observed $\hat{c}_{1}, \hat{s}_{1}, \hat{c}_{3}, \hat{s}_{3}$. Below we summarize the results.

\subsubsection{Case I.-Approximate Viewing Angles $\hat{\Gamma}_{\mathrm{obs}}, \hat{i}$ from a Fit to the Geometry of the Orbit}

The observed radial velocity is expanded with respect to the angle $\hat{\psi}$, which is the angle in the plane of the deprojected ring and which is zero at the line of nodes. This definition is very similar to that of the true angle $\psi$. The resulting expression for the velocity field becomes

$$
\begin{gathered}
v_{r}=\hat{c}_{1} \cos \hat{\psi}+\hat{s}_{1} \sin \hat{\psi}+\hat{c}_{3} \cos 3 \hat{\psi}+\hat{s}_{3} \sin 3 \hat{\psi}, \\
\hat{c}_{1}=\left[1-\left(\frac{3}{4}-a\right) \epsilon_{R} \cos 2 \phi_{\mathrm{obs}}\right] v_{c} \sin i, \\
\hat{s}_{1}=\left\{\left[\frac{5-q^{2}}{4\left(1-q^{2}\right)}-a\right] \epsilon_{R} \sin 2 \phi_{\mathrm{obs}}\right\} v_{c} \sin i, \\
\hat{c}_{3}=\left[\left(a-\frac{1}{4}\right) \epsilon_{R} \cos 2 \phi_{\mathrm{obs}}\right] v_{c} \sin i, \\
\hat{s}_{3}=\left[\left(\frac{1}{4}-a\right) \epsilon_{R} \sin 2 \phi_{\mathrm{obs}}\right] v_{c} \sin i,
\end{gathered}
$$

where $q=\cos \hat{i}$. It is the apparent axis ratio of the projected orbit. The above expressions are valid only for small $\epsilon_{R}$ and large $i$. As expected, if the orbit is intrinsically circular, all terms $\hat{s}_{1}, \hat{c}_{3}$, and $\hat{s}_{3}$ will be zero. If the ellipticity is nonzero, then we can measure significant $\cos \hat{\psi}, \sin \hat{\psi}, \cos 3 \hat{\psi}$, and $\sin 3 \hat{\psi}$ terms.

We show an example of a deprojected velocity field in Figure 2. The velocity field is dominated by the $\cos \hat{\psi}$ term, which is the term typical of a circular orbit. The next largest term is the $\sin \hat{\psi}$ term, which also produces the "minor axis" rotation. A measurement of the $\sin \hat{\psi}$ term is a very sensitive measurement of $\epsilon_{R} \sin 2 \phi_{\mathrm{obs}}$. This term is the difference in elongation of the potential along the axes $\phi=\phi_{\text {obs }}+\pi / 2$ and $\phi=\phi_{\text {obs }}-\pi / 2$. This coefficient was labeled $s_{\psi}$ by Kuijken \& Tremaine (1994). It is zero when the potential (and the ellipse) are viewed down their long or short axis. The complementary term $\epsilon_{R} \cos 2 \phi_{\text {obs }}$ can be derived from the $\cos 3 \hat{\psi}$ coefficient in the fit to the velocity field. Unfortunately, the maximum amplitude of this term is lower by at least a factor of 5 than the maximum amplitude of the $\sin \hat{\psi}$ term (for $a \sim 0$ ). Thus, in many cases,
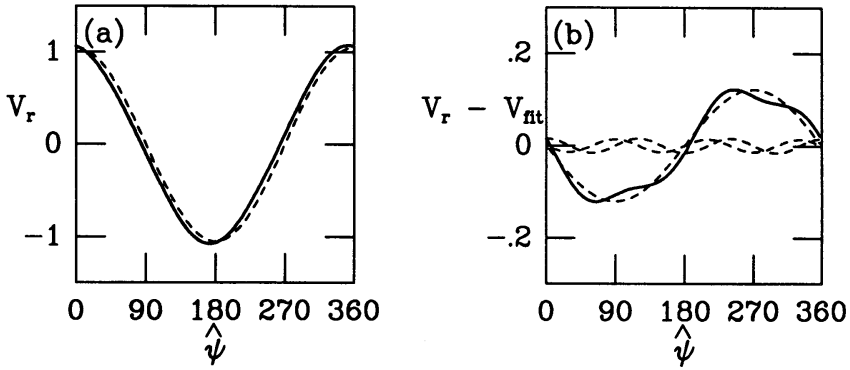

FIG. 2.-An example of the observed radial velocity along a projected elliptic orbit. The potential has an ellipticity of 0.1 in the plane of the ring. A circular orbit is fitted to the projected ellipse, and the velocity field is expanded in harmonics defined by this coordinate system. Panel $(a)$ shows the radial velocity as a function of the angle $\hat{\psi}$, which is zero at the line of nodes. The dashed curve is the best-fitting circular orbit. For such an orbit, the radial velocity is proportional to $\cos \psi$. The additional terms are caused by the ellipticity of the potential. Panel (b) shows the residuals from the circular orbit fit. The dashed curves show how this residual can be expanded into the sin $\hat{\psi}$, $\cos 3 \hat{\psi}$ and $\sin 3 \hat{\psi}$ harmonics. The $\sin \hat{\psi}$ term is by far the largest term caused by the ellipticity of the potential.

$\epsilon_{R} \sin 2 \phi_{\mathrm{obs}}$ will be much better determined than $\epsilon_{R} \cos 2 \phi_{\mathrm{obs}}$, and it may be better to view these as the two independent terms which are measured by the fit.

\subsubsection{Case II.-Approximate Viewing Angles from a Fit of a Circular Velocity Field}

Second, we can determine the approximate viewing angles from a fit of a circular velocity field to the observed velocity field, ignoring the geometry of the ring. In the Appendix all necessary expressions are given. We find that the approximate inclination $\hat{i}$ is equal to the true inclination $i$ for the case of a flat rotation curve. Thus, in this special case, a fit of a circular velocity field to the velocity field of an elliptic orbit produces the correct inclination of the elliptic orbit. This result was used earlier by Franx \& de Zeeuw (1992). The implication is that galaxy inclinations from a fit to the velocity field are much less influenced by a noncircular potential than inclinations derived from galaxy photometry.

The velocity field can be expressed as

$$
v_{r}=\hat{c}_{1} \cos \hat{\psi}+\hat{s}_{1} \sin \hat{\psi}+\hat{s}_{3} \sin 3 \hat{\psi},
$$

$$
\begin{aligned}
\hat{c}_{1}= & v_{c} \sin i\left(1-\epsilon_{R} \cos 2 \phi_{\mathrm{obs}}\right), \\
\hat{s}_{1}= & v_{c} \sin i\left\{1-a-\frac{\left(1-q^{2}\right)\left[3 q^{2}+1-2 a\left(q^{2}+1\right)\right]}{\left(3 q^{2}+1\right)^{2}+\left(1-q^{2}\right)^{2}}\right\} \\
& \times \epsilon_{R} \sin 2 \phi_{\mathrm{obs}}, \\
\hat{c}_{3}= & 0, \\
\hat{s}_{3}= & v_{c} \sin i\left\{-a-\frac{\left(1-q^{2}\right)\left[3 q^{2}+1-2 a\left(q^{2}+1\right)\right]}{\left(3 q^{2}+1\right)^{2}+\left(1-q^{2}\right)^{2}}\right\} \\
& \times \epsilon_{R} \sin 2 \phi_{\mathrm{obs}},
\end{aligned}
$$

where $q=\cos \hat{i}$. This quantifies Teuben's (1991) result, who numerically analyzed noncircular velocity fields. As expected, all terms except the $\cos \hat{\psi}$ term disappear if the orbit is circular. If the orbit is noncircular, then both terms $\hat{c}_{3}$ and $\hat{s}_{3}$ are proportional to $\epsilon_{R} \sin 2 \phi_{\text {obs }}$. 
If we take $v_{c} \sin i, \epsilon_{R} \cos 2 \phi_{\text {obs }}$, and $\epsilon_{R} \sin 2 \phi_{\text {obs }}$ as independent parameters, then it becomes clear that they cannot be determined uniquely from equation (8), if the $\hat{c}_{1}, \hat{s}_{1}, \hat{s}_{3}$ terms are measured. For most practical purposes, however, it will be safe to assume that $\epsilon_{R}$ is small, and then, $v_{c} \sin i$ and $\epsilon_{R} \sin 2 \phi_{\text {obs }}$ can be well measured. The term $\epsilon_{R} \cos 2 \phi_{\text {obs }}$ remains undetermined. Hence, for small $\epsilon_{R}$ it is impossible to measure the total ellipticity of the potential from the velocity field alone.

\subsection{The Final Method for Measuring the Elongation of the Potential}

The above results show that the first method is the most sensitive method to measure the ellipticity of the potential. In this case, the geometry of the ring is used to derive the approximate viewing angles under the assumption that the orbit is circular. The velocity field is expanded in harmonics, $v_{r}=$ $\sum \hat{c}_{n} \cos n \hat{\psi}+\hat{s}_{n} \sin n \hat{\psi}$. The $\hat{s}_{1}$ term is directly proportional to $\epsilon_{R} \sin 2 \phi_{\text {obs }}$, and the $\hat{c}_{3}$ and $\hat{s}_{3}$ terms are proportional to $\epsilon_{R} \cos 2 \phi_{\text {obs }}$ and $\epsilon_{R} \sin 2 \phi_{\text {obs }}$ (but with a smaller constant of proportionality). It follows that a definite measurement of these three terms is sufficient to determine $\epsilon_{R}, \phi_{\mathrm{obs}}$. It $\epsilon_{R}$ is significant, then the slope of the rotation curve, $\alpha$, can also be estimated. Equation (8) gives the constants of proportionality for the epicyclic orbits. In principle, equation (8) can be solved to give $\phi_{\text {obs }}, \alpha, \epsilon_{R}$, and $v_{c}$ explicitly as a function of $\hat{c}_{1}, \hat{s}_{1}, \hat{c}_{3}$, and $\hat{s}_{3}$. In practice, this will require ultraprecise measurements, mainly because $\alpha$ is poorly constrained.

It is more convenient to assume a value for $\alpha$ and then to derive the remaining parameters. We give the general expressions in the Appendix. For a flat rotation curve we have $\alpha=0$ and obtain

$$
\begin{aligned}
\epsilon_{R}\left|\sin 2 \phi_{\mathrm{obs}}\right| & =4\left[\frac{\left(1-q^{2}\right)}{\left(5-q^{2}\right)}\right]\left|\frac{\hat{s}_{1}}{\hat{c}_{1}}\right|, \\
\epsilon_{R} & =4 \frac{\left(\hat{c}_{3}^{2}+\hat{s}_{3}^{2}\right)^{1 / 2}}{\hat{c}_{1}} .
\end{aligned}
$$

The potential shape can be measured very accurately with this method. If we can measure the individual terms $\hat{c}_{i}, \hat{s}_{i}$ to $1 \%$, then $\epsilon_{R} \sin 2 \phi_{\text {obs }}$ can be determined very accurately from the $\hat{s}_{1}$ term (to better than $1 \%$ ). The coefficients $\hat{s}_{3}, \hat{c}_{3}$ can also be used to estimate the shape of the potential. An error of $1 \%$ in each implies an error of 0.06 in the overall ellipticity. Although much larger than the error in $\epsilon_{R} \sin 2 \phi_{\text {obs }}$, this still gives useful results. Furthermore, as we will show in $\S 4$, the terms $\hat{c}_{i}, \hat{s}_{i}$ can be determined to high accuracy from high signal-to-noise ratio H idata.

We now define several statistical tests which can be used to test certain hypotheses concerning the shape of the potential. We can use two criteria to rule out the hypothesis that the potential is circular, one based on the $\hat{s}_{1}$ coefficient, and the other on the $\hat{s}_{3}, \hat{c}_{3}$ terms. We can rule out a circular potential at the $95 \%$ confidence level if

$$
\left|\hat{s}_{1}\right|>1.96 \sigma\left(\hat{s}_{1}\right),
$$

or

$$
\hat{s}_{3}^{2}+\hat{c}_{3}^{2}>2.45 \sigma\left(\hat{s}_{3}\right)^{2}
$$

We have assumed that the errors are distributed like a Gaussian, and that $\sigma\left(\hat{s}_{3}\right)=\sigma\left(\hat{c}_{3}\right)$. Similarly, we can calculate an upper limit to $\epsilon_{R} \sin 2 \phi_{\text {obs }}$, and the ellipticity of the potential $\epsilon_{R}$. To this end, we calculate upper limits to $\hat{s}_{1}$ and $\sqrt{\hat{s}_{3}^{2}+\hat{c}_{3}^{2}}$ under the assumption that the probability distribution of the true parameters is a Gaussian around the observed values. The $95 \%$ confidence upper limits for each quantity are given by

$$
\begin{aligned}
\epsilon_{R}\left|\sin 2 \phi_{\mathrm{obs}}\right| & <\frac{\left(5-q^{2}\right)}{4\left(1-q^{2}\right)} \frac{\left[\left|\hat{s}_{1}\right|+1.96 \sigma\left(\hat{s}_{1}\right)\right]}{\left|\hat{c}_{1}\right|}, \\
\epsilon_{R} & <4 \frac{\lim \left(\sqrt{\hat{s}_{3}^{2}+\hat{c}_{3}^{2}}\right)}{\left|\hat{c}_{1}\right|} .
\end{aligned}
$$

We performed various numerical simulations to test the reliability of these upper limits, and found that these tests work very satisfactorily.

\section{21 CENTIMETER LINE OBSERVATIONS OF IC 2006}

The original Schweizer et al. (1989) observations were done as a detection experiment, with low angular and velocity resolution. The main motivation for reobserving the galaxy was to get much better velocity resolution, in order to derive the shape of the dark halo, The observations were made in 1989 October with the Very Large Array (Napier, Thompson, \& Ekers 1983) with the array in its $1 \mathrm{~km}$ (D) configuration, but with an extended north arm $(3 \mathrm{~km})$ to compensate for the low declination of the source. The observations were spread over 4 days, resulting in a total of $24 \mathrm{hr}$ on source. A total bandwidth of $3.125 \mathrm{MHz}$ was used, centered at a heliocentric velocity of $1390 \mathrm{~km} \mathrm{~s}^{-1}$ (optical definition). Online Hanning smoothing was employed, after which every other channel was discarded. This results in a set of 63 independent channels, separated by $10.4 \mathrm{~km} \mathrm{~s}^{-1}$, for each of the two polarizations. The flux density scale is based on an assumed value of $15.81 \mathrm{Jy}$ for $3 \mathrm{C} 48$ at our sky frequency. The field center was at the optical center of IC $2006, \alpha_{1950}=3^{\mathrm{h}} 52^{\mathrm{m}} 35.9, \quad \delta_{1950}=$ $-36^{\circ} 06^{\prime} 47^{\prime \prime} .4$ (Schweizer et al. 1989).

Standard calibration procedures were followed. A uniform weighted cube was made and a continuum image was formed by averaging a set of line-free channels on either side of the band. This image was subtracted from the cube. The channels showing line emission were CLEANed and finally the cube was convolved to a circular beam $\left(40^{\prime \prime}\right)$. The rms noise in the channel maps is $0.8 \mathrm{mJy}$ per beam.

The main result of these new observations is that the neutral hydrogen ring around IC 2006 is filled and that its velocity field is remarkably regular. The surface density distribution is rather smooth. Figure 3 shows the individual channel maps centered on the systemic velocity of IC 2006. The velocity of each channel is given in the upper right-hand corner, and the small cross marks the optical center of the galaxy. The channel maps form a textbook example of a ring in circular rotation.

The integrated hydrogen image is shown in Figure 4. The regularity of the ring is again striking. It appears filled with gas at our resolution. There is some structure along the ring. The flux distribution is depressed toward the southeast. The noise is always smaller than the lowest contour, so the features along the ring are real. Although the ring is very narrow, it is slightly resolved. We address this issue in $\S 4$.

Figure 5 presents the intensity-weighted velocity field. This is our main result; the velocity field is remarkably regular. The mean flux-weighted velocity of the ring is $1389 \mathrm{~km} \mathrm{~s}^{-1}$. This agrees with the velocity of $1385 \pm 7 \mathrm{~km} \mathrm{~s}^{-1}$ derived by Schweizer et al. (1989). 


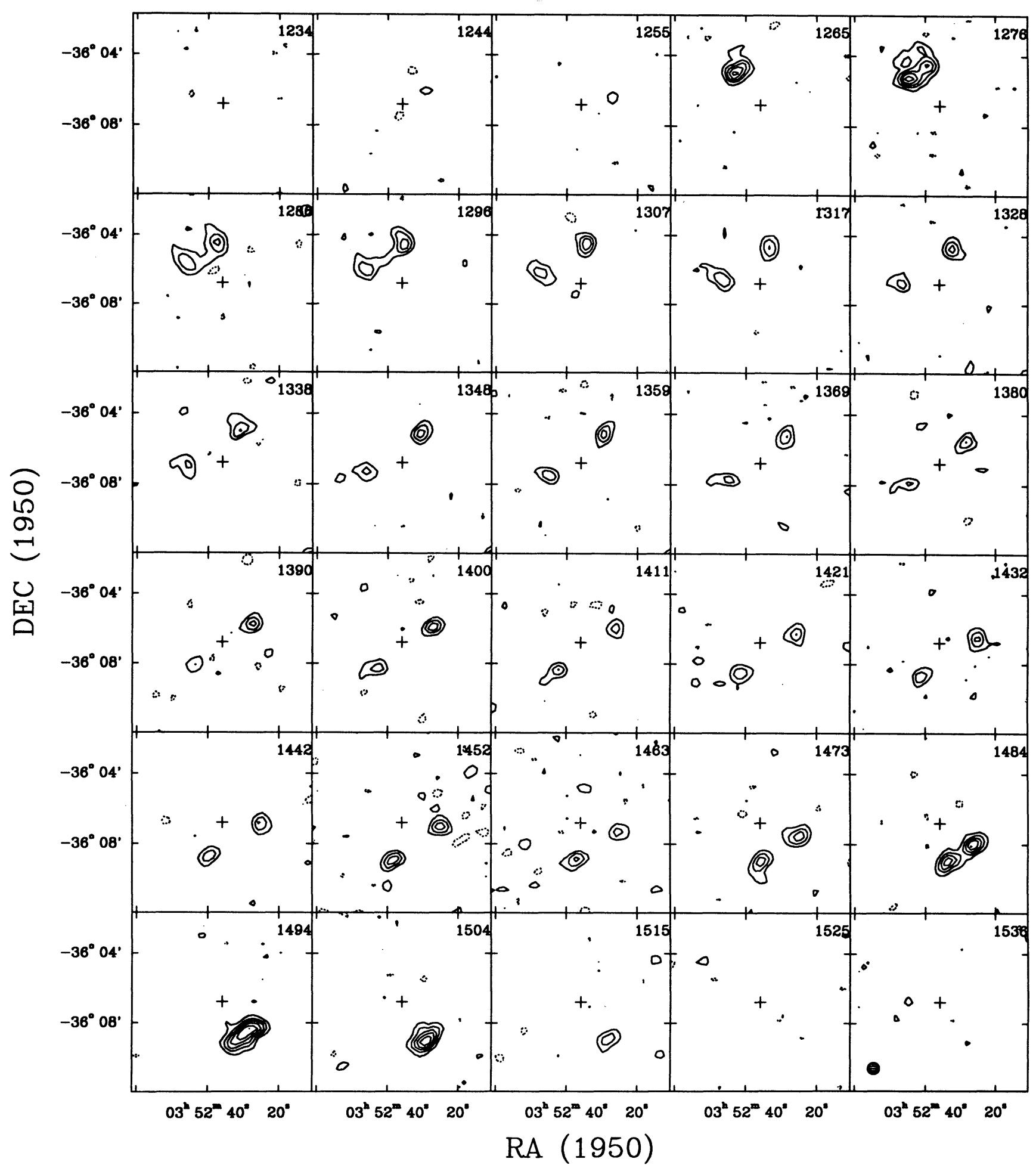

FIG. 3.-Individual channel maps of the $\mathrm{H}$ I distribution in IC 2006. The contour interval is $2 \mathrm{mJy}$ per beam. Negative contours are dotted. The heliocentric radial velocity $\left(\mathrm{km} \mathrm{s}^{-1}\right)$ of each channel is indicated at the top right in each frame. The plus signs mark the optical center of IC 2006. The hatched circle in the lower right-hand frame shows the size of the synthesized beam (HPBW).

\section{MODELING OF THE H I RING}

\subsection{Ring Geometry}

The first step in the modeling is to fit an ellipse to the $\mathbf{H}_{\mathbf{I}}$ flux map. We tested a variety of algorithms for this purpose and decided to use the following procedure. Assume a center $\left(x_{0}, y_{0}\right)$, position angle $\tilde{\Gamma}_{\text {ring }}$, and major and minor axes of the ellipse $a$ and $b$. Hence, the ellipse can be written as

$$
\frac{\tilde{x}^{2}}{a^{2}}+\frac{\tilde{y}^{2}}{b^{2}}=1
$$

where $\tilde{x}$ and $\tilde{y}$ are a suitably defined coordinate system with origin at the center of the ellipse, and coordinate axes parallel 


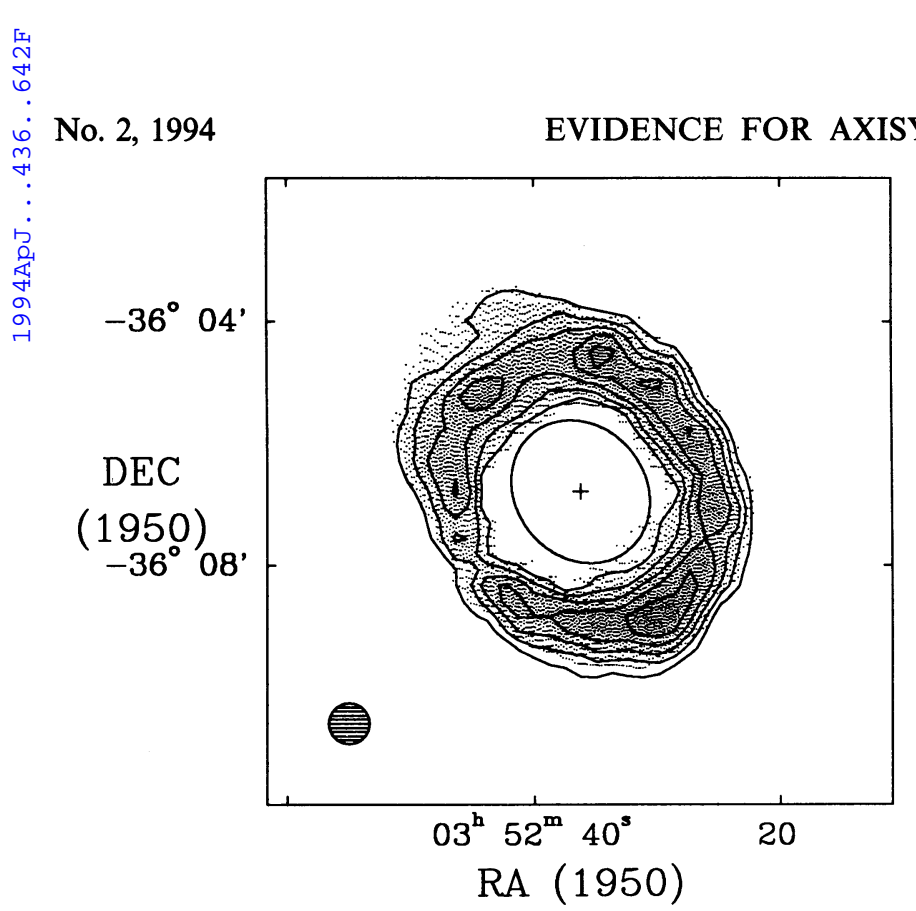

Fig. 4.- Map of total $\mathrm{H}$ I distribution in IC 2006. The contour interval is $3.4 \times 10^{19}$ atoms cm$~_{-2}$. The regularity of the ring is striking. The plus marks the center of the optical galaxy, and the ellipse shows the ellipticity and position angle of the outer well-defined isophotes of the optical galaxy (taken from Schweizer et al. 1989). The ellipticity and position angle of this ellipse appear to agree well with those of the $\mathrm{H}$.

to the major and minor axis of the ellipse. We calculate the values of $x_{0}, y_{0}, a, b, \tilde{\Gamma}_{\text {ring }}$ which minimized the residual

$$
\chi^{2}=\sum_{i}\left[\left(\tilde{x}_{i}-a \cos \hat{\psi}_{i}\right)^{2}+\left(\tilde{y}_{i}-b \sin \hat{\psi}_{i}\right)^{2}\right] \text { Flux }_{i}
$$

where the angle $\hat{\psi}$ is given by

$$
\tan \hat{\psi}=\frac{\tilde{y} / b}{\tilde{x} / a} .
$$

For a perfectly thin ellipse, this residual is zero. The minimum is found by a straightforward linearized least-squares fitting routine. No more than 10 iterations were needed to find the final solution.

An advantage of this approach is that it produces error estimates. The error results directly from the linearized leastsquares fit. Furthermore, the shape of the fitted ellipse is not strongly influenced by any irregular flux distribution along the ellipse. Any method employing the moments of the flux distribution would be strongly influenced by such an irregular flux distribution. Finally, we can estimate the width of the ellipse from $\chi^{2}$. We verified with numerical simulations that this method gave unbiased estimates of the ring parameters.

Table 1 presents the results of the ellipse fitting procedure. We repeated the ellipse fitting routine on maps in which pixels below a given flux limit were omitted. We chose two flux limits

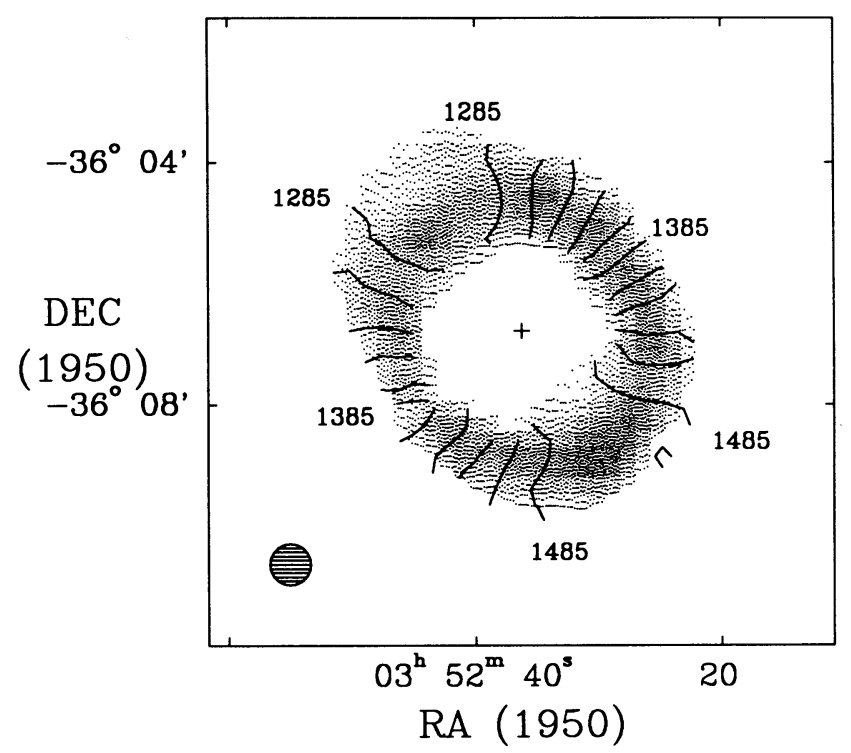

FIG. 5.-The intensity-weighted mean $\mathrm{H}$ I velocities in IC 2006. The contours are spaced by $20 \mathrm{~km} \mathrm{~s}^{-1}$, and some of the velocities are marked. The systemic velocity of the galaxy is $1385 \mathrm{~km} \mathrm{~s}^{-1}$. The gray-scale map represents the total $\mathrm{H}$ I flux distribution.

such that one-third and $2 / 3$ of the pixels fell below the limits. The results are given in the second and third columns of Table 1. It is evident that the axis lengths of the ellipses vary somewhat with higher limits, but that the ellipticity and position angle remain very stable. This gives us confidence that ellipticity and position angle are well determined.

The radial distribution of the flux is presented in Figure 6. The total flux in elliptical bins is plotted in Figure $6 a$. Figure $6 b$ shows the cumulative flux inside elliptical annuli as a function of the major axis of the annulus. The radial coordinate is normalized to the size of the fitted ellipse. Also plotted are the expected distributions for an infinitely narrow ring, convolved with the beam width. The ring is clearly more extended. We are able to produce the observed distribution very well with a ring which has a Gaussian flux distribution in the radial direction. The dispersion in radius is 0.12 times the major axis length.

The ring is deprojected under the assumption that the ring is circular. The implied inclination angle is $31^{\circ}$. Figure $6 c$ shows the flux distribution as a function of the angle $\hat{\psi}$ in the plane of the deprojected ring. The flux distribution is not quite regular, and there is a depression at $\hat{\psi} \approx 90^{\circ}$. This depression is also apparent in the flux map in Figure 4.

\subsection{Ring Kinematics}

Figure $7 a$ shows the velocities of the ring as a function of the angle $\hat{\psi}$. The velocities are resampled into bins of $\Delta \hat{\psi}=18^{\circ}$.

TABLE 1

\begin{tabular}{|c|c|c|c|}
\hline \multirow[b]{2}{*}{ PARAMETER } & \multicolumn{3}{|c|}{ FluX } \\
\hline & $\frac{>1}{\left(\mathrm{mJy} \mathrm{beam}^{-1} \mathrm{~km} \mathrm{~s}^{-1}\right)}$ & $\begin{array}{c}>70 \\
\left(\mathrm{mJy} \mathrm{beam}^{-1} \mathrm{~km} \mathrm{~s}^{-1}\right)\end{array}$ & $\stackrel{>157}{>}\left(\mathrm{mJy} \mathrm{beam}^{-1} \mathrm{~km} \mathrm{~s}^{-1}\right)$ \\
\hline $\begin{array}{l}a \\
\epsilon_{\text {ring }} \\
\Gamma_{\text {ring }} \ldots \ldots \ldots \ldots \ldots \ldots \ldots \ldots \ldots \ldots \ldots\end{array}$ & $\begin{array}{l}144^{\prime \prime} \pm 2^{\prime \prime} \\
0.14 \pm 0.02 \\
34^{\circ} \pm 5^{\circ}\end{array}$ & $\begin{array}{l}141^{\prime \prime} \pm 2^{\prime \prime} \\
0.13 \pm 0.02 \\
35^{\circ} \pm 5^{\circ}\end{array}$ & $\begin{array}{l}139^{\prime \prime} \pm 2^{\prime \prime} \\
0.13 \pm 0.03 \\
36^{\circ} \pm 5^{\circ}\end{array}$ \\
\hline
\end{tabular}

GeOMETRY OF THE RING 

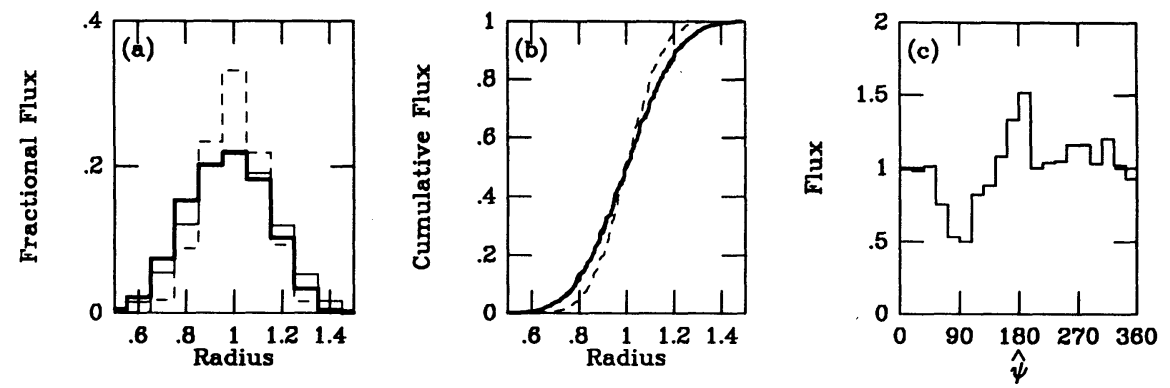

FIG. 6.-The flux distribution measured in coordinates defined by the best-fitting ellipse. (a) Flux distribution of the ellipse as a function of normalized radius. For each point, the normalized radius is calculated as the ratio of the observed to predicted distance to the center of the ellipse. The predicted distance is the distance to the fitted ellipse measured along the line through the center and the observed point. The dashed line shows the prediction for an infinitely narrow ring, convolved with the beam. The thin, drawn, line shows the prediction for a ring with a relative intrinsic width of 0.12 . (b) As $(a)$, but now showing the cumulative flux distribution. (c) The $\mathbf{H}$ I flux as a function of the angle $\hat{\psi}$. The data have been summed into independent bins. The distribution is quite regular but shows a dip near $\hat{\psi}=90^{\circ}$. This distribution is predicted to be flat, for a regular, circular orbit.

Because of this resampling, the noise is nearly independent, and the error estimates from the harmonic fit are reliable.

It is striking how regular the velocity field is. We expand the velocities in the form described in $\S 2$.

$$
v_{r}=\sum_{n=0}^{4} \hat{c}_{n} \cos \hat{\psi}+\hat{s}_{n} \sin \hat{\psi},
$$

and find

$$
\begin{array}{ll}
\hat{c}_{1}=113.5 \pm 0.4 \mathrm{~km} \mathrm{~s}^{-1}, & \hat{s}_{1}=0.6 \pm 0.5 \mathrm{~km} \mathrm{~s}^{-1} \\
\hat{c}_{2}=1.1 \pm 0.4 \mathrm{~km} \mathrm{~s}^{-1}, & \hat{s}_{2}=0.6 \pm 0.4 \mathrm{~km} \mathrm{~s}^{-1}, \\
\hat{c}_{3}=-0.3 \pm 0.4 \mathrm{~km} \mathrm{~s}^{-1}, & \hat{s}_{3}=-0.2 \pm 0.4 \mathrm{~km} \mathrm{~s}^{-1}
\end{array}
$$

The errors are the formal errors of the harmonic fit. We again test the robustness of the fit by omitting pixels with fluxes lower than a certain limit. We choose the same limits as for the geometrical fit and repeat the velocity fit. The coefficients change slightly with increasingly higher flux cutoff. The largest relative variation is in the $\hat{s}_{1}$ term, which varies between -2.5 and $0.6 \mathrm{~km} \mathrm{~s}^{-1}$. This is mostly due to variations in the position angle of the ellipse. The third-order terms are always below 1 $\mathrm{km} \mathrm{s}^{-1}$. We remark that the amplitude $\hat{c}_{1}$ of the rotation is somewhat lower than the value of $122 \pm 5 \mathrm{~km} \mathrm{~s}^{-1}$ estimated by eye by Schweizer et al. (1989).
The residuals of the velocity fit after subtraction of the $\cos \hat{\psi}$ term are shown in Figure $7 b$. It is reassuring to see how small the residuals are. The residuals at $\hat{\psi}=90^{\circ}$ are related to the dip in the flux at that angle. The finite spatial resolution of the observations causes the flux from neighboring regions to unduly influence the velocity at this location. The strongest residuals are the $\hat{c}_{2}, \hat{s}_{2}$ terms. These terms indicate that the center of the velocity field does not coincide with the center of ellipse. We repeated the ellipse fit but fixed the center to be the optical center of the galaxy. The ellipticity and position angle of the best-fitting ellipse changed very little. The resulting harmonic fit to the velocity fit gives much smaller residuals; see Figure $7 c$. The $\hat{c}_{2}, \hat{s}_{2}$ are now smaller than $0.2 \mathrm{~km} \mathrm{~s}^{-1}$, which shows that the kinematic center coincides with the optical center of the galaxy.

The observations imply that the potential is nearly circular in the plane of the ring. We have measured no significant $\hat{s}_{1}, \hat{c}_{3}$, and $\hat{s}_{3}$ terms. Table 2 presents the resulting values of $\epsilon_{R} \sin \phi_{\mathrm{obs}}$ and $\epsilon_{R}$, which have been calculated from the harmonic expansion terms by equations (9) and (12). The second column gives the values when only the errors from the velocity fit are taken into account. None of the measurements indicate an ellipticity which deviates significantly from 0 . An axisymmetric potential is completely consistent with the results. Table 2 also gives upper limits to the ellipticity. These limits are chosen such that
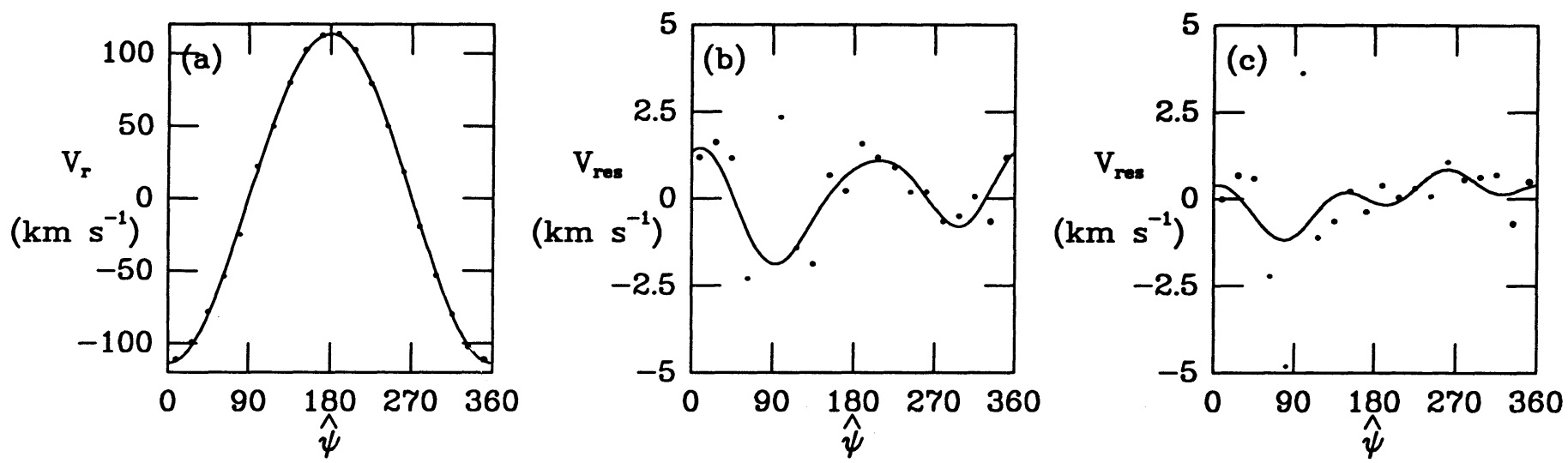

FIG. 7.-The observed velocity field along the ring. The measured velocities have been averaged into 20 independent bins. $(a) v_{r}$ as a function of $\hat{\psi}$. The size of the points is indicative of the $\mathrm{H}$ I flux at that point. The curve is the circular orbit fit. (b) The residuals from the circular orbit fit as a function of $\hat{\psi}$. The residuals are dominated by the $\cos 2 \hat{\psi}$ and $\sin 2 \hat{\psi}$ terms, which are caused by a slight offset between the geometric center of the ring, and the kinematic center. (c) The residuals from the circular orbit fit if the center of the ellipse is fixed to the center of the optical galaxy. The relatively large residuals near $\hat{\psi}=90^{\circ}$ are caused by beam-smearing effects near the "gap" in the ring. The $\sin \hat{\psi}, \cos 3 \hat{\psi}$, and $\sin 3 \hat{\psi}$ terms are all smaller than $1 \mathrm{~km} \mathrm{~s}^{-1}$. 
TABLE 2

Measurement of the Shape of the Potential

\begin{tabular}{ccccc}
\hline \hline & & \multicolumn{2}{c}{$\begin{array}{c}\text { ERRORS FROM Both ELLIPSE } \\
\text { FIT AND VELOCITY FIT }\end{array}$} \\
\cline { 3 - 4 } PARAMETER & $\begin{array}{c}\text { ERLOCITY FIT } \\
\text { Detection }\end{array}$ & Detection & Upper Limit \\
\hline$\epsilon_{R} \sin 2 \phi_{\text {obs }} \ldots \ldots \ldots$ & $0.001 \pm 0.001$ & $0.001 \pm 0.019$ & $<0.038$ \\
$\epsilon_{R} \cdots \cdots \cdots \cdots \cdots \cdots$ & $0.012 \pm 0.015$ & $0.012 \pm 0.026$ & $<0.050$ \\
\hline
\end{tabular}

the probability that the true values of $\epsilon_{R} \sin 2 \phi_{\text {obs }}, \epsilon_{R}$ are higher than the upper limits are smaller than 0.05 .

The errors which are used in the second column are based on the errors in the velocity field only. These errors do not incorporate the errors in the ellipticity and position angle. It turns out that a variation in the position angle of $5^{\circ}$ produces a much larger variation in $\hat{s}_{1}$ than the formal error in $\hat{s}_{1}$. One might argue that such errors are only bound to give a higher ellipticity for the ring, and not a smaller. Although this is undoubtedly the case, if we want to derive a reliable error estimate, or a reliable $95 \%$ confidence limit, then we cannot ignore this effect. The third and fourth columns in Table 2 incorporate all the sources of errors and are therefore more reliable.

\subsection{Numerical Simulations}

In order to test the reliability of the result, we have performed Monte Carlo simulations of our full procedure. We simulate the ring as a collection of discrete $\mathrm{H}$ I clouds. The clouds are put on an elliptic closed orbit in a potential with arbitrary elongation. The radial distribution is Gaussian and set to reproduce the observed width of the ring $(\S 3.2)$. The number of clouds and the ellipticity of the potential are varied. We compute 1000 realizations for each of these models and derive estimates of the ellipticity and upper limits for each realization.

We find that the upper limits are reliable for intrinsic ellipticities smaller than 0.06 . This gives us further confidence in the result. If the ring is given no intrinsic width, then the errors are overestimated, and the upper limits are also overestimates, which makes us err on the safe side.

The main uncertainty in the analysis is the physical noise in the ring, i.e., the fact that the material may not be distributed in a narrow, homogeneous ring. Our simulations give us confidence that we have estimated the uncertainty due to this effect in a reasonable way. We note however, that this uncertainty relates directly to the underlying assumption throughout this work that the ring is in equilibrium. We cannot rule out the possibility that the ring is not in equilibrium and that our measurement of very low ellipticity is incorrect. This is possible if the shape of the ring deviates from the shape of a closed orbit. We consider this unlikely, however, in view of the fact that the velocity field is extremely regular. A definite test can only be made by observations of a large sample of galaxies with narrow rings.

\section{MODELS OF THE LIGHT AND MASS DISTRIBUTION}

\subsection{Luminous Material}

The very small elongation of the potential derived in the previous section implies that the viewing angles of the ring are well determined by the standard assumption that the ring is intrinsically circular. We next assume that the ring lies in the symmetry plane of the galaxy. Although it is not possible to verify this assumption independently, it appears to be reasonable, given the regular velocity field of the ring, and the small difference in position angle and ellipticity between the ring and the outer parts of the galaxy.

Under these assumptions, the inclination of the galaxy is the same as that of the ring, $31^{\circ}$. We deproject the surface photometry of Schweizer et al. (1989), assuming that the galaxy is axisymmetric, and that the surfaces of constant density are stratified on spheroids. We applied the formulas which are valid for galaxies which are stratified on similar spheroids (e.g., Stark 1977) to find the intrinsic ellipticity $\epsilon_{\text {gal }}(r)$. The resulting ellipticity profile is shown in Figure 8 . The ellipticity is seen to increase to very high values of $0.6-0.7$ in the outer parts. Such ellipticities are never observed for "normal" ellipticals, and we therefore conclude that the galaxy probably has a strong disk. By itself, this conclusion is not very surprising. Many galaxies classified as ellipticals contain weak or strong disks (see, e.g., Carter 1987; Capaccioli 1990; Bender 1988; Rix \& White 1990). The fact that IC 2006 follows an $r^{1 / 4}$ law reasonably well is not very decisive, as the low central surface brightness of a face-on disk makes it very hard to detect.

The isophotal twist found by Schweizer et al. (1989) indicates that IC 2006 is not perfectly axisymmetric. We deproject the observed ellipticity and position angle profile with the formalism of Stark (1977) for triaxial ellipsoids. We fix the inclination and vary the azimuthal viewing angle $\phi_{\mathrm{obs}}$. The deviation from axisymmetry is smaller than $5 \%$ for most values of $\phi_{\text {obs }}$. This gives us confidence that the axisymmetric modeling is appropriate.

\subsection{Mass Distribution}

Finally, we construct a mass model to compare to the available data. First, we construct an axisymmetric model based on the surface photometry, using the techniques designed by Binney, Davies, \& Illingworth (1990) and adapted by van der Marel (1991). The last author kindly made available his software.

We introduce a small modification to this software. We reproject the galaxy to an inclination of $90^{\circ}$ (edge-on), under the

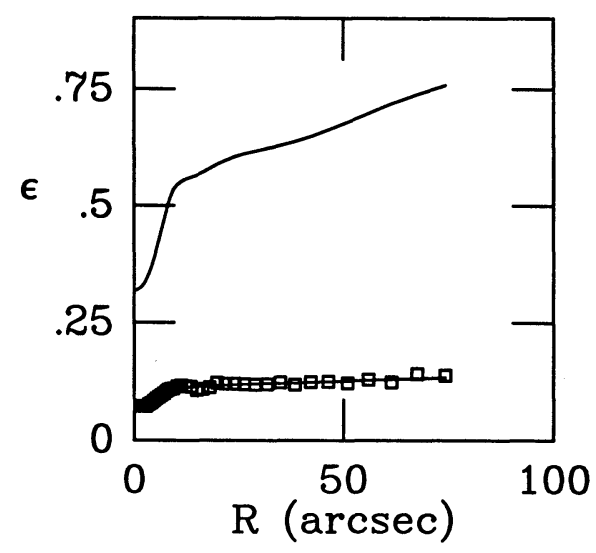

Fig. 8.-Deprojection of the light distribution, under the assumption that the $\mathrm{H}_{\mathrm{I}}$ ring defines the viewing angles, and that the galaxy is axisymmetric. The upper drawn line gives the curve of intrinsic ellipticity. This line has been smoothed to reduce the noise. The points indicate the observed ellipticity. The bottom curve shows the reprojection of the upper curve of intrinsic ellipticity. The large intrinsic ellipticity of $0.6-0.7$ at large radii indicates that the galaxy has a disk in the outer parts. 
assumption that the density distribution is axisymmetric. This procedure improves the deprojection algorithm. We assume first that the $M / L$ ratio is constant. The central velocity dispersion predicted by the model can be compared to the measured dispersion of $128 \mathrm{~km} \mathrm{~s}^{-1}$ by Davies et al. (1987). The resulting mass-to-light ratio $M / L=5.6 M_{\odot} / L_{\odot}$, which agrees very well with the value estimated by Schweizer et al. (1989) using the core-fitting formula.

The resulting curve of circular velocity as function of radius is given in Figure 9. The circular velocity has a peak of $242 \mathrm{~km}$ $\mathrm{s}^{-1}$ at $r=11^{\prime \prime}$ and declines gently further out. The measured rotation velocity of the $\mathrm{H}$ I gas is also indicated in the figure and is clearly inconsistent with the predicted value based on a constant $M / L$. Thus we confirm the radial gradient in the $M / L$ ratio found earlier by Schweizer et al. (1989). Our model implies that $M_{\text {tot }} / M_{\text {lum }}=3.2$ inside $6.5 R_{e}$, where $M_{\text {lum }}$ is the luminous mass, assuming a constant $(M / L)_{\text {lum }}$.

The similarity between the circular velocity at $v\left(r=11^{\prime \prime}\right)=$ $242 \mathrm{~km} \mathrm{~s}^{-1}$ and $v\left(r=120^{\prime \prime}\right)=221 \pm 14 \mathrm{~km} \mathrm{~s}^{-1}$ is surprising, and suggests that the circular velocity is nearly constant at radii larger than $11^{\prime \prime} \sim 0.5 R_{e}$. Thus the mass distribution in IC 2006 is probably very well described by an $r^{-2}$ density profile, and it is very similar to the mass distribution in spiral galaxies. We construct a second model with a very flat curve of circular velocity. We add a density component of the form $\rho=\rho_{0} /$ $\left[1+\left(r / r_{0}\right)^{2}\right]$, with $r_{0}=33^{\prime \prime}$ and an asymptotic circular velocity of $259 \mathrm{~km} \mathrm{~s}^{-1}$. The resulting curve of circular velocity is shown in Figure 9. It is constant to $4 \mathrm{~km} \mathrm{~s}^{-1}$ outside of $11^{\prime \prime}$ from the center. The predicted circular velocity at the ring is slightly too high, $248 \mathrm{~km} \mathrm{~s}^{-1}$, as compared to $221 \pm 14 \mathrm{~km} \mathrm{~s}^{-1}$. The uncertainty in the model value is fairly large, since it is based only on a central velocity dispersion.

Our central $M / L$ ratio is sensitive to (small) anisotropies in the velocity distributions. Long-slit kinematical data taken at several position angles will help to test the assumptions required for the modeling. Our present conclusion is that the data implies a strong increase in the $M / L$ ratio with radius and is consistent with a curve of circular velocity which is nearly flat at large radii. The logarithmic gradient between the model value at $r=11^{\prime \prime}$ and the observed value at $r=144^{\prime \prime}$ is -0.05 .

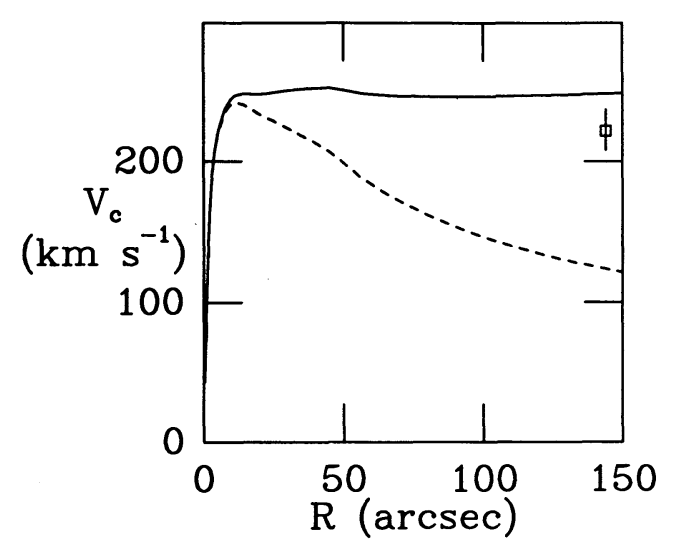

FIG. 9.-The model curves of predicted circular velocity $v_{c}$. The drawn line shows $v_{c}$ under the assumption that the $M / L$ ratio is constant. The $M / L$ is derived from the central velocity dispersion of the stars. The circular velocity at the ring is shown in the upper right, and it exceeds the predicted circular velocity by a factor of 1.8 . The dashed line shows the predicted circular velocity curve when a halo is added which results into a flat rotation curve. It agrees much better with the circular velocity indicated by the ring.

\section{DISCUSSION}

We have shown that the E/S0 galaxy IC 2006 has a significant dark matter component within the radius of the ring $(r=$ $144^{\prime \prime}=6.5 R_{e}$ ). This confirms the previous result by Schweizer et al. (1989). The radial change in $M / L$ ratio is about 3.2. Our models indicate that the curve of circular velocity is nearly flat outside $11^{\prime \prime}$. The potential is nearly axisymmetric.

We have developed a sensitive method to measure the elongation of the potential in the plane of the ring. This extends earlier analyses by Binney (1978) and Teuben (1991). A circular orbit is fitted to the geometry of the ring, and the velocity field of a ring is expended into harmonic terms. We present expressions which relate these harmonic terms directly to the viewing angles, and to the intrinsic parameters of the ring. The expressions are valid for small $\epsilon_{R}$, the ellipticity of the ring. We find that the ellipticity of the ring can be fully determined in this way. When only the velocity field is used, and not the geometry of the ring, then the ellipticity cannot be fully measured. In this case, one obtains a measurement of $\epsilon_{R} \sin 2 \phi_{\text {obs }}$, where $\phi_{\text {obs }}$ is one of the viewing angles.

We note that complementary expressions have been derived by Kuijken \& Tremaine (1994), who considered the observable effects of elongated orbits in our Galaxy.

When we apply our method to new data on the ring in IC 2006 , we find that it is nearly circular $\left(\epsilon_{\text {pot }}=0.012 \pm 0.026\right)$. This result is surprising, given the ease by which dissipationless simulations of halo formation produce triaxial halos (Frenk et al. 1988; White \& Ostriker 1990; Dubinski \& Carlberg 1991). However, it extends the previous result by Franx and de Zeeuw (1992) that the potentials of spiral galaxies have small systematic elongation in the plane of the disk. The discrepancy between the current results and the dissipationless simulations may be caused by the infall of baryonic matter in the dark matter halo, as suggested by recent simulations of Katz and Gunn (1991) and Navarro \& White (1994). These authors find that the halos are more nearly oblate axisymmetric when baryonic matter is included in the simulations.

Models for the light distribution show that IC 2006 is probably very flat in the outer parts, suggesting that it has a disk. It may therefore be better classified as an S0. The presence of a dark matter halo is further proof for the continuity between early-type galaxies and late-type spirals and suggests that both may contain sizeable fractions of "dark matter," and were formed in a qualitatively similar way.

The current sample of galaxies with measured halo shapes is still small (e.g., Sackett \& Sparke 1990). The method presented here can be applied easily to other systems with regular gas rings. It should be possible to measure the flattening and circularity of halos by analysis of high signal-to-noise ratio, highresolution kinematic observations of (polar) ring galaxies. The best results are obtained when the shape and velocity field of the ring can be used. Unfortunately, the number of known galaxies with regular gas rings is small.

Furthermore, the method can be used to analyze the velocity fields of spiral galaxies. The first analyses by Bosma (1978) did not show large effects due to noncircular potentials, but this result has not been quantified yet.

We thank the NRAO for generous allocation of observing time. The VLA of the National Radio Astronomy Observatory is operated by Associated Universities, Inc. under a cooperative agreement with the National Science Foundation. 
We thank Joanna Lees for stimulating discussions and communication of results prior to publication. It is a pleasure to thank Roeland van der Marel for making available his software. M. F. was funded by Hubble Fellowship grant HF-
1016.01.91A. This work has been supported in part by NSF grants AST 89-17744 and AST 90-23254 to Columbia University. The comments of the referee, James Binney, have helped to improve the paper.

\section{APPENDIX}

\section{HARMONIC ANALYSIS OF THE VELOCITY FIELD OF A RING}

\section{A1. EPICYCLIC APPROXIMATION}

The orbit is assumed to lie in the $(x, y)$-plane and to be elongated along the $y$-axis. To first order in the ellipticity $\epsilon_{\text {pot }}$ of the potential, standard epicyclic theory (Gerhard \& Vietri 1986; Binney \& Tremaine 1987) shows that the orbit is given by

$$
R=R_{0}\left(1-\frac{1}{2} \epsilon_{R} \cos 2 \Omega_{0} t\right), \quad \phi=\Omega_{0} t+\frac{1}{4}\left(\epsilon_{R}+\epsilon_{v}\right) \sin 2 \Omega_{0} t,
$$

where $R$ and $\phi$ are the usual polar coordinates defined by $x=R \cos \phi, y=R \sin \phi, t$ is time, and $\Omega_{0}$ is the circular frequency of the orbit with radius $R_{0}$. For a potential with constant ellipticity and circular velocity $v_{c} \propto R^{\alpha}$ the coefficients $\epsilon_{R}$ and $\epsilon_{v}$ are

$$
\epsilon_{R}=\frac{1+\alpha}{1-\alpha} \epsilon_{\mathrm{pot}}, \quad \epsilon_{v}=\frac{2 \epsilon_{R}}{1+\alpha} .
$$

The components $v_{R}$ and $v_{\phi}$ of the orbital velocity at the point $(R, \phi)$ are given by

$$
v_{R}=v_{c} \epsilon_{R} \sin 2 \phi, \quad v_{\phi}=v_{c}\left(1+\frac{1}{2} \epsilon_{v} \cos 2 \phi\right),
$$

and $v_{c}$ is the circular velocity evaluated at $R_{0}$.

The orbit is observed along a line of sight defined by the polar coordinates $\left(i, \phi_{\mathrm{obs}}\right)$. The resulting velocity field along a single orbit is given by

$$
v_{r}=\left[v_{\phi} \sin \left(\phi-\phi_{\mathrm{obs}}\right)-v_{R} \cos \left(\phi-\phi_{\mathrm{obs}}\right)\right] \sin i .
$$

We introduce a coordinate $\psi=\phi-\phi_{\text {obs }}-\pi / 2$. This coordinate is zero along the apparent major axis of the projected circular orbit $\boldsymbol{R}=$ constant. Substituting equation (A3) into the general expression (A4), eliminating $\phi$ in favor of $\psi$, and expressing the result in a harmonic series in $\psi$ then produces

$$
v_{r}=v_{c} \sin i\left\{\left[1-(1-a) \epsilon_{R} \cos 2 \phi_{\mathrm{obs}}\right] \cos \psi+\left[(1-a) \epsilon_{R} \sin 2 \phi_{\mathrm{obs}}\right] \sin \psi+\left(a \epsilon_{R} \cos 2 \phi_{\mathrm{obs}}\right) \cos 3 \psi-\left(a \epsilon_{R} \sin 2 \phi_{\mathrm{obs}}\right) \sin 3 \psi\right\},
$$

and $a=\frac{1}{2} \alpha /(1+\alpha)$. Expressions valid for a general rotation curve $v_{c}=v_{c}(R)$ can be derived in a similar way.

\section{A2. GENERAL DEPROJECTION UNDER THE WRONG VIEWING ANGLES}

When the viewing angles for deprojection $\hat{\Gamma}_{\text {obs }}, \hat{i}$ are not equal to the true viewing angles $\Gamma, i$, then $v_{r}$ will again be given by an expression of the form of (A5), but with slightly different coefficients. It is straightforward to express the original terms $\cos n \psi, \sin n \psi$ in harmonic expansions $\sum_{i} a_{i} \cos i \hat{\psi}+b_{i} \sin i \hat{\psi}$. These can be used to derive the general expression for the velocity field

$$
v_{r}=\hat{c}_{1} \cos \hat{\psi}+\hat{s}_{1} \sin \hat{\psi}+\hat{c}_{3} \cos 3 \hat{\psi}+\hat{s}_{3} \sin 3 \hat{\psi}+\hat{c}_{5} \cos 5 \hat{\psi}+\hat{s}_{5} \sin 5 \hat{\psi}+\cdots,
$$

where

$$
\begin{aligned}
& \hat{c}_{1}=c_{1}\left(1-\frac{1}{4} \frac{\delta q}{q}\right)+\delta \Gamma_{\mathrm{obs}} \frac{1}{4}\left(\frac{3}{q}+q\right) s_{1}-\frac{3}{4} \frac{\delta q}{q} c_{3}+\frac{3}{4} \delta \Gamma_{\mathrm{obs}}\left(\frac{1}{q}-q\right) s_{3}, \\
& \hat{s}_{1}=s_{1}\left(1+\frac{1}{4} \frac{\delta q}{q}\right)-\delta \Gamma_{\mathrm{obs}} \frac{1}{4}\left(3 q+\frac{1}{q}\right) c_{1}-\frac{3}{4} \delta \Gamma_{\mathrm{obs}}\left(\frac{1}{q}-q\right) c_{3}-\frac{3}{4} \frac{\delta q}{q} s_{3}, \\
& \hat{c}_{3}=c_{3}+\frac{1}{4} \frac{\delta q}{q} c_{1}+\delta \Gamma_{\mathrm{obs}} \frac{1}{4}\left(\frac{1}{q}-q\right) s_{1}+\delta \Gamma_{\mathrm{obs}} \frac{3}{2}\left(q+\frac{1}{q}\right) s_{3}, \\
& \hat{s}_{3}=s_{3}-\frac{1}{4} \delta \Gamma_{\mathrm{obs}}\left(\frac{1}{q}-q\right) c_{1}+\frac{1}{4} \frac{\delta q}{q} s_{1}-\frac{3}{2} \delta \Gamma_{\mathrm{obs}}\left(q+\frac{1}{q}\right) c_{3}, \\
& \hat{c}_{5}=\frac{3}{4} \frac{\delta q}{q} c_{3}+\frac{3}{4} \delta \Gamma_{\mathrm{obs}}\left(\frac{1}{q}-q\right) s_{3}, \\
& \hat{s}_{5}=\frac{3}{4} \delta \Gamma_{\mathrm{obs}}\left(\frac{1}{q}-q\right) c_{3}+\frac{3}{4} \frac{\delta q}{q} s_{3},
\end{aligned}
$$


and we have defined $q$ by $q=\cos i$ and $\delta q=\hat{q}-q$. It follows that $q$ equals the axis ratio of the orbit only if it is circular. For small deviations from a circular orbit, $s_{1}, c_{3}$, and $s_{3}$ are small quantities, and so are $\delta q$ and $\delta \Gamma_{\text {obs. }}$ Ignoring second-order terms, equations (A7) then reduce to

$$
\begin{aligned}
& \hat{c}_{1}=c_{1}\left(1-\frac{1}{4} \frac{\delta q}{q}\right) \\
& \hat{s}_{1}=s_{1}-\delta \Gamma_{\text {obs }} \frac{1}{4}\left(3 q+\frac{1}{q}\right) c_{1}, \\
& \hat{c}_{3}=c_{3}+\frac{1}{4} \frac{\delta q}{q} c_{1}, \\
& \hat{s}_{3}=s_{3}-\frac{1}{4} \delta \Gamma_{\text {obs }}\left(\frac{1}{q}-q\right) c_{1} .
\end{aligned}
$$

We now use these equations to calculate the expected values of $\hat{c}_{1}, \hat{s}_{1}, \hat{c}_{3}$, and $\hat{s}_{3}$, for two different ways of determining approximate values of $q$ and $\Gamma_{\text {obs }}$.

\section{A3. DEPROJECTION USING THE GEOMETRY OF THE RING}

If the geometry of the ring is used to derive the approximate viewing angle, assuming the ring is intrinsically circular, then to first order $\delta q$ and $\delta \Gamma_{\text {obs }}$ are given by

$$
\frac{\delta q}{q}=-\epsilon_{R} \cos 2 \phi_{\mathrm{obs}}, \quad \delta \Gamma_{\mathrm{obs}}=-\frac{q \epsilon_{R} \sin 2 \phi_{\mathrm{obs}}}{1-q^{2}} .
$$

These expressions are valid for small $d q / q$ and $\delta \Gamma_{\text {obs }}$ and assume that $\sin ^{2} i \gg \epsilon_{R}$, so that the ring is not nearly face-on. The resulting expressions for the measured harmonic terms along the deprojected ring are

$$
\begin{aligned}
& \hat{c}_{1}=\left[1-\left(\frac{3}{4}-a\right) \epsilon_{R} \cos 2 \phi_{\mathrm{obs}}\right] v_{c} \sin i, \\
& \hat{s}_{1}=\left\{\left[\frac{5-q^{2}}{4\left(1-q^{2}\right)}-a\right] \epsilon_{R} \sin 2 \phi_{\mathrm{obs}}\right\} v_{c} \sin i, \\
& \hat{c}_{3}=\left[\left(a-\frac{1}{4}\right) \epsilon_{R} \cos 2 \phi_{\mathrm{obs}}\right] v_{c} \sin i, \\
& \hat{s}_{3}=\left[\left(\frac{1}{4}-a\right) \epsilon_{R} \sin 2 \phi_{\mathrm{obs}}\right] v_{c} \sin i,
\end{aligned}
$$

This completes the proof of equation (8).

We can now derive a velocity amplitude $\hat{v}_{c}$ with the usual assumption that the orbit is circular. This is related to the true circular velocity by

$$
\hat{v}_{c}=\frac{\hat{c}_{1}}{\sin \hat{i}}=v_{c}\left\{1-\left[\frac{3+q^{2}}{4\left(1-q^{2}\right)}-a\right] \epsilon_{R} \cos 2 \phi_{\mathrm{obs}}\right\} .
$$

The apparent radius of the ring $\hat{R}$ is not equal to the true radius of the ring $R$, but is given by

$$
\hat{R}=R\left(1+\frac{1}{2} \epsilon_{R} \cos 2 \phi_{\mathrm{obs}}\right) \text {. }
$$

\section{A4. DEPROJECTION USING KINEMATIC FIT}

Let us first consider the general case of a fit of a circular velocity field to an observed velocity field. The angles $\hat{\Gamma}_{\text {obs }}$ and $\hat{i}$ will be chosen to minimize the residuals from the circular model $v_{r}=\hat{c}_{1} \cos \hat{\psi}$. We can expand the velocity field into the harmonics $\hat{c}_{i}$, $\hat{s}_{i}$. If these measured coefficients are independent, then the residual will be proportional to the sum $\hat{s}_{1}^{2}+\sum_{i>1}\left(\hat{c}_{i}^{2}+\hat{s}_{i}^{2}\right)$. Equation (A8) can be used to calculate the change in these coefficients if $\hat{\Gamma}_{\text {obs }}$ and $q \equiv \cos \hat{i}$ are changed by a small amount $\delta \Gamma_{\text {obs }}$ and $\delta q$. If we ignore the higher order terms, then the residual from the circular orbit fit can be written as

$$
\chi^{2}=\left[\hat{s}_{1}-\frac{1}{4} \delta \Gamma_{\mathrm{obs}}\left(3 q+\frac{1}{q}\right) c_{1}\right]^{2}+\left(\hat{c}_{3}+\frac{1}{4} \frac{\delta q}{q} c_{1}\right)^{2}+\left[\hat{s}_{3}-\frac{1}{4} \delta \Gamma_{\mathrm{obs}}\left(\frac{1}{q}-q\right)\right]^{2} .
$$

It is straightforward to calculate the best-fitting values of $\delta \Gamma_{\text {obs }}$ and $\delta q$

$$
\begin{aligned}
\delta q & =-\frac{4 q \hat{c}_{3}}{\hat{c}_{1}+\hat{c}_{3}}, \\
\delta \Gamma_{\text {obs }} & =4 q \frac{\left(3 q^{2}+1\right) \hat{s}_{1}+\left(1-q^{2}\right) \hat{s}_{3}}{\left[\left(3 q^{2}+1\right)^{2}+\left(1-q^{2}\right)^{2}\right]\left(\hat{c}_{1}+\hat{c}_{3}\right)} .
\end{aligned}
$$


These expressions for $\delta \Gamma_{\text {obs }}$ and $\delta q$ will generally be used in an iterative way. One calculates these values at each iteration step from the harmonic terms, updates the viewing angles, and recalculates the harmonic terms. Although circular velocity fields have been fitted to H I radio maps since the early 1970s (Warner, Wright, \& Baldwin 1973), we have not been able to find these rather simple expressions in the literature.

We can now apply these equations if a circular model is fitted to an elliptic orbit. We start by taking the correct viewing angles. The harmonic terms are given by equation (A5). Equation (A14) gives the first-order change in inclination and position angle to achieve the best circular fit. It is straightforward to derive

$$
\begin{aligned}
\delta q & =-4 a \epsilon_{R} \cos 2 \phi_{\mathrm{obs}}, \\
\delta \Gamma_{\mathrm{obs}} & =4 \frac{q\left[3 q^{2}+1-2 a\left(q^{2}+1\right)\right]}{\left[\left(3 q^{2}+1\right)^{2}+\left(1-q^{2}\right)^{2}\right]} \epsilon_{R} \sin 2 \phi_{\mathrm{obs}} .
\end{aligned}
$$

We deproject the velocity field with the new viewing angles $\hat{\Gamma}_{\text {obs }}$ and $\hat{i}$, given by equation (A15). The resulting velocity field in the new coordinate $\hat{\psi}$ is

$$
v_{r}=\hat{c}_{1} \cos \hat{\psi}+\hat{s}_{1} \sin \hat{\psi}+\hat{s}_{3} \sin 3 \hat{\psi}
$$

where

$$
\begin{aligned}
& \hat{c}_{1}=v_{c} \sin i\left(1-\epsilon_{R} \cos 2 \phi_{\mathrm{obs}}\right), \\
& \hat{s}_{1}=v_{c} \sin i\left\{1-a-\frac{\left(1-q^{2}\right)\left[3 q^{2}+1-2 a\left(q^{2}+1\right)\right]}{\left(3 q^{2}+1\right)^{2}+\left(1-q^{2}\right)^{2}}\right\} \epsilon_{R} \sin 2 \phi_{\mathrm{obs}}, \\
& \hat{c}_{3}=0 \\
& \hat{s}_{3}=v_{c} \sin i\left\{-a-\frac{\left(1-q^{2}\right)\left[3 q^{2}+1-2 a\left(q^{2}+1\right)\right]}{\left(3 q^{2}+1\right)^{2}+\left(1-q^{2}\right)^{2}}\right\} \epsilon_{R} \sin 2 \phi_{\mathrm{obs}} .
\end{aligned}
$$

For a flat rotation curve $(a=0)$, these expressions simplify considerably (see eq. [8]). Equation $(\mathrm{A} 15)$ implies that $\delta q=\delta i=0$. In other words, a fit with a circular velocity field produces the correct inclination for a galaxy with a flat rotation curve. The best-fitting position angle will still be offset from the true position angle. We can define the apparent deprojected circular velocity $\hat{v}_{c}$ by

$$
\hat{v}_{c}=\hat{c}_{1} / \sin \hat{i}=v_{c}\left(1-\epsilon_{R} \cos 2 \phi_{\mathrm{obs}}\right) \text {. }
$$

Again, the apparent deprojected circular velocity will not be equal to the true circular velocity. For the case considered here of a flat rotation curve, the circular velocity estimated from the fit of a circular velocity is nearer to the true circular velocity than the circular velocity estimated from a geometric fit to the ring ( $\$$ A3 of the Appendix).

Bender, R. 1988, A\&A, 193, L7

Binney, J. J. 1978, MNRAS, 183, 779

Binney, J. J., Davies, R. L., \& Illingworth, G. D. 1990, ApJ, 361, 78

Binney, J. J., \& Tremaine, S. D. 1987, Galactic Dynamics (Princeton: Princeton Univ. Press)

Bosma, A. 1978, Ph.D. thesis, Univ. of Groningen

Capaccioli, M. 1990, in IAU Symp. 147, Structure and Dynamics of Elliptical Galaxies, ed. P. T. de Zeeuw (Dordrecht: Kluwer), 47

Carter, D. 1987, ApJ, 312, 514

Davies, R. L., Burstein, D., Dressler, A., Faber, S. M., Lynden-Bell, D., Terlevich, R. J., \& Wegner, G. 1987, ApJS, 64, 81

de Zeeuw, P. T. 1992, in Morphological and Physical Classification of Galaxies, ed. G. Busarello, M. Capaccioli, \& G. Longo (Dordrecht: Kluwer), 139

Dubinski, J., \& Carlberg, R. G. 1991, ApJ, 378, 496

Franx, M., \& de Zeeuw, P. T. 1992, ApJ, 392, L47

Frenk, C. S., White, S. D. M., Davis, M., \& Efstathiou, G. 1988, ApJ, 327, 507

Gerhard, O. E., \& Vietri, M. 1986, MNRAS, 223, 377

\section{REFERENCES}

Katz, N., \& Gunn, J. E. 1991, ApJ, 377, 365

Kent, S. M. 1990, in Evolution of the Universe of Galaxies, ed. R. G. Kron (ASP Conf. Ser., 10), 109

Kuijken, K., \& Tremaine, S. D. 1994, ApJ, 421, 178

Napier, P. J., Thompson, A. R., \& Ekers, R. D. 1983, Proc. IEEE, 71, 1295

Navarro, J., \& White, S. D. M. 1994, MNRAS, in press

Rix, H.-W., \& White, S. D. M. 1990, ApJ, 362, 52

Sackett, P., \& Sparke, L. S. 1990, ApJ,, 361, 408

Schweizer, F., van Gorkom, J. H., \& Seitzer, P. 1989, ApJ, 338, 770

Stark, A. A. 1977, ApJ, 213, 368

Teuben, P. J. 1991, in Warped Disks and Inclined Rings around Galaxies, ed. S. Casertano, P. Sackett, \& F. Briggs (Cambridge: Cambridge Univ. Press) 40

Tohline, J. E., Simonson, G. F., \& Caldwell, N. 1982, ApJ, 252, 92 van der Marel, R. P. 1991, MNRAS, 253, 710

Warner, P. J., Wright, M. C. H., \& Baldwin, J. E. 1973, MNRAS, 163, 163

White, S. D. M., \& Ostriker, J. P. 1990, ApJ, 349, 22 